\title{
Publisher Correction: Voices of biotech leaders
}

Katrine Bosley, Charlotte Casebourn, Priscilla Chan, Janice Chen, Michael Chen, George Church, John Cumbers, Tomas de Wouters, Heather Dewey-Hagborg, Xavier Duportet, Abasi Ene-Obong, Arturo Elizondo, Jeremy Farrar, Bill Gates, Francesco Gatto, Sebastian Giwa, Jernej Godec, Silvia Gold, Emily LeProust, Jeantine Lunshof, Eddie Martucci, Michelle McMurray Heath, Jason Mellad, Veronika Oudova, Neri Oxman, Aviv Regev, Sarah Richardson, Christopher Thomas Scott, Jake Sherkow, Leah Sibener, Teresa Tarragó, Sharon Terry, J. Craig Venter, Spin Wang, Sajith Wickramasekara, Hakim Yadi, Luhan Yang and Bowen Zhao

Correction to: Nature Biotechnology https://doi.org/10.1038/s41587-021-00941-4, published online 10 June 2021.

In the version of this article initially published, an author name was given as Abasi Ene Abong. The correct name is Abasi Ene-Obong. Also, the affiliation for Sebastian Giwa was given as Elevian, Pagliuca Harvard Life Lab, Allston, MA, USA. The correct affiliations are Biostasis Research Institute, Berkeley, CA, USA; Sylvatica Biotech, North Charleston, SC, USA; and Humanity Bio, Kensington, CA, USA. An affiliation for Jeantine Lunshof was given as Department of Genetics, Harvard Medical School, Boston, MA, USA. The correct affiliation is Wyss Institute for Biological Engineering, Harvard University, Boston, MA, USA. The errors have been corrected in the PDF and HTML versions of the article.

Published online: 21 July 2021

https://doi.org/10.1038/s41587-021-01000-8

(c) Springer Nature America, Inc. 2021

\section{Publisher Correction: Questions swirl around failures of disease-modifying Huntington's drugs}

Cormac Sheridan

Correction to: Nature Biotechnology https://doi.org/10.1038/s41587-021-00955-y, published online 10 June 2021.

In the version of this article initially published, WVE-120102 and WVE-120101 were described in the main text and WVE-003 in Table 1 as $2^{\prime}$-deoxy and 2'-O-methoxyethyl phosphorothioate triester ASOs. WVE-120102 and WVE-120101 are allele-selective stereodefined $2^{\prime}$-deoxy and 2'-O-methyl phosphorothioate and phosphorodiester chimeric ASOs. WVE-003 is an alelle-selective, stereopure ASO (structure not publicly available) that promotes RNase-H-mediated degradation of a mutant mRNA HTT species containing an undisclosed SNP. Also, WVE-120102 and WVE-120101 were described as targeting SNP1 (rs362307) and SNP2 (rs362331), respectively; the two SNPs here were transposed. Finally, they were described as being given in phase 1 testing; the trial was phase $1 / 2$. The errors have been corrected in the PDF and HTML versions of the article.

Published online: 26 July 2021

https://doi.org/10.1038/s41587-021-01017-z

(c) The Author(s), under exclusive licence to Springer Nature America, Inc. 2021

\section{Publisher Correction: COVID-19 long haulers}

\section{Charles Schmidt}

Correction to: Nature Biotechnology https://doi.org/10.1038/s41587-021-00984-7, published online 13 July 2021.

In this article an interviewee was identified as Dennis Charney, a neurologist at Mount Sinai. Dennis Charney is a psychiatrist at Mount Sinai. However, the interviewee was Alexander Charney, another psychiatrist at Mount Sinai. The error has been corrected in the print, PDF and HTML versions of the article. 